\title{
Penfigoide ampolloso asociado a vildagliptina
}

\section{Bullous pemphigoid associated with vildagliptin}

\author{
Campbell-Silva $S E^{1}$, de la Cruz-Esparza $L^{2}$, Vásquez-Arias $F^{2}$.
}

\author{
${ }^{1}$ Médico internista, servicio de medicina interna, Clínica Mediláser, \\ Florencia, Caquetá. \\ ${ }^{2}$ Médico general, servicio de medicina interna, Clínica Mediláser, \\ Florencia, Caquetá. \\ Autor de correspondencia: Santiago Emilio Campbell-Silva \\ Correo electrónico: santiago.campbell@gmail.com \\ Fecha de recepción: 10/09/2019 \\ Fecha de aceptación: 11/03/2020
}

\section{Resumen}

La prevalencia de la diabetes mellitus tipo 2 continúa aumentando constantemente a causa de la creciente esperanza de vida de la población y los inhibidores de la dipeptidil-peptidasa IV, medicamentos orales sin riesgo de hipoglucemia y de efecto neutro con respecto al peso, se convierten en una opción terapéutica para este grupo de personas mayores. No obstante, estos fármacos se han asociado a reacciones cutáneas graves, entre ellas el penfigoide ampolloso. Por tanto, debido a la morbilidad y mortalidad asociadas a esta afección, es necesaria la sensibilización de los profesionales de la salud para que conozcan este vínculo y estén atentos ante cualquier reacción adversa cutánea de estos medicamentos en las poblaciones de riesgo. Presentamos dos casos de penfigoide ampolloso asociados al uso de vildagliptina.

Palabras clave: diabetes mellitus tipo 2, inhibidores de la dipeptidil-peptidasa IV, penfigoide ampolloso.

\section{Abstract}

The prevalence of type 2 diabetes mellitus continues to increase steadily as more people live longer, and dipeptidyl peptidase-IV inhibitors, oral medications with no risk of hypoglycemia and neutral with respect to weight, become a therapeutic option for this group of elderly. However, dipeptidyl peptidase-4 inhibitors have been associated with severe skin reactions including bullous pemphigoid, and due to the morbidity and mortality associated with this condition, it is necessary to sensitize health professionals to know this link and be alert to any adverse skin reaction of these medications in at risk populations. We present two cases of bullous pemphigoid associated with vildagliptin.

Keywords: Bullous pemphigoid; Dipeptidyl peptidase-IV inhibitors; Diabetes mellitus, type 2.

\section{Introducción}

El penfigoide ampolloso (PA) es el tipo más común de las enfermedades ampollosas autoinmunitarias subepidérmicas (1). Tradicionalmente se considera como una enfermedad de la población de edad avanzada. La edad media de presentación oscila entre los 66 y los 83 años en diferentes cohortes en todo el mundo (2, 3). La incidencia aumenta exponencialmente con la edad, de 190 a 312 casos por millón y por año en personas mayores de 70 años (3). Sin embargo, en las últimas dos décadas se ha notado un aumento importante en la incidencia de 1,9 a 4,3 veces (3), aumento que debe generar mayor conciencia en el cuerpo médico por el alto riesgo de desarrollar PA secundario al uso de medicamentos, sobre todo en este grupo etario que tiene generalmente polifarmacia. El PA rara vez se encuentra en individuos menores de 50 años, y las tasas de incidencia informadas suelen ser inferiores a 0,5 casos por millón de habitantes en esta categoría de edad (3). En cuanto al sexo existe una preponderancia del femenino en la mayoría de los estudios, relación mujer-hombre entre 1,04 y 5,1 (3).

La diabetes mellitus es una patología frecuente en la población general y en particular en la población geriátrica, con un crecimiento constante en los últimos años debido a la mayor sobrevida. Los personas mayores tienen mayor riesgo de desarrollar reacciones adversas farmacológicas (hipoglucemia) en comparación con los más jóvenes debido a los cambios fisiológicos asociados a la edad, al número alto de comorbilidades que suelen presentar y a la polifarmacia, ocasionando que la farmacocinética y farmacodinámica de los medicamentos se altere notablemente Los inhibidores de la dipeptidil-peptidasa IV (iDPP-IV) son una buena elección para este grupo de pacientes debido a su administración oral, el bajo riesgo de hipoglucemia, el efecto neutro sobre el peso y tienen bajo índice de interacción con otras drogas teniendo en cuenta la existencia de la polifarmacia. Sin embargo, existe evidencia creciente 
que los iDPP-IV están implicados en el desarrollo del PA $(4,5)$. Presentamos dos casos relacionados con el uso de estos medicamentos; hasta donde conocemos, en la bibliografía colombiana es el primer informe.

\section{Presentación de los casos}

Paciente masculino de 72 años (caso 1) y femenina de 83 años (caso 2), ambos con diagnósticos de diabetes mellitus tipo 2 (DM2) de varios años de evolución. Para el control de la DM2, el paciente masculino utilizaba glimepirida y metformina; posteriormente le prescribieron vildagliptina más metformina en forma combinada y se mantuvo la administración de glimepirida. Durante 16 meses utilizó estos medicamentos antes de la aparición de las lesiones en piel. Por su parte, la paciente utilizaba insulina glargina y metformina y posteriormente, en forma combinada, vildagliptina más metformina durante 9 meses antes de la aparición de las lesiones. Ambos pacientes no tenían antecedentes ni datos sugestivos de procesos neoplásicos, infecciosos, autoinmunes ni de enfermedades neurológicas o neurodegenerativas. A excepción de unos eosinófilos periféricos de 530 células por microlitro (caso 2), los demás estudios solicitados no mostraron alteraciones: radiografía de tórax, ecografía abdominal, hemograma, proteína C-reactiva, AST, ALT, creatinina, serología VDRL, TSH, T3, T4, alfafetoproteína, antígeno carcinoembrionario, CA-125, calcitonina, deshidrogenasa láctica y CA 19-9.

Los pacientes iniciaron lesiones cutáneas pruriginosas papulares, que posteriormente se convirtieron en ampollas tensas de diámetros variables, principalmente en el tronco y las extremidades. Cuando estas lesiones se rompían, dejaban erosiones húmedas (Figuras 1 y 2). Las imágenes del primer caso fueron tomadas por el mismo paciente al inicio de la enfermedad, quien rompía las ampollas para automedicarse con crema de ácido fusídico.

La primera biopsia de piel del caso 1 fue compatible, según el informe, con pitiriasis liquenoide crónica (microscopia: ligera acantosis de la epidermis y leve infiltrado linfocitario en la dermis superior con histiocitos y depósito de pigmento pardo). Por prescripción recibió diversos antibióticos orales, varios corticoides tópicos, tacrólimus y lubricación sin resultados satisfactorios y con recidivas frecuentes. Después de 1 año del proceso fue valorado por el dermatopatólogo, quien hizo el diagnóstico de penfigoide ampolloso, el cual comprobó mediante biopsia (microscopia: en la dermis se observa gran ampolla subepidérmica con un infiltrado predominantemente eosinófilo, que se organiza a lo largo de la membrana basal) y sugirió estudios de inmunofluorescencia, que no fueron practicados. Hubo una mejoría notoria con la administración de prednisona oral y azatioprina; actualmente no presenta le-

Figura 1. Caso 1. Aspecto de las lesiones una vez que las ampollas se han roto. La coloración amarillenta es por la crema utilizada por el paciente.
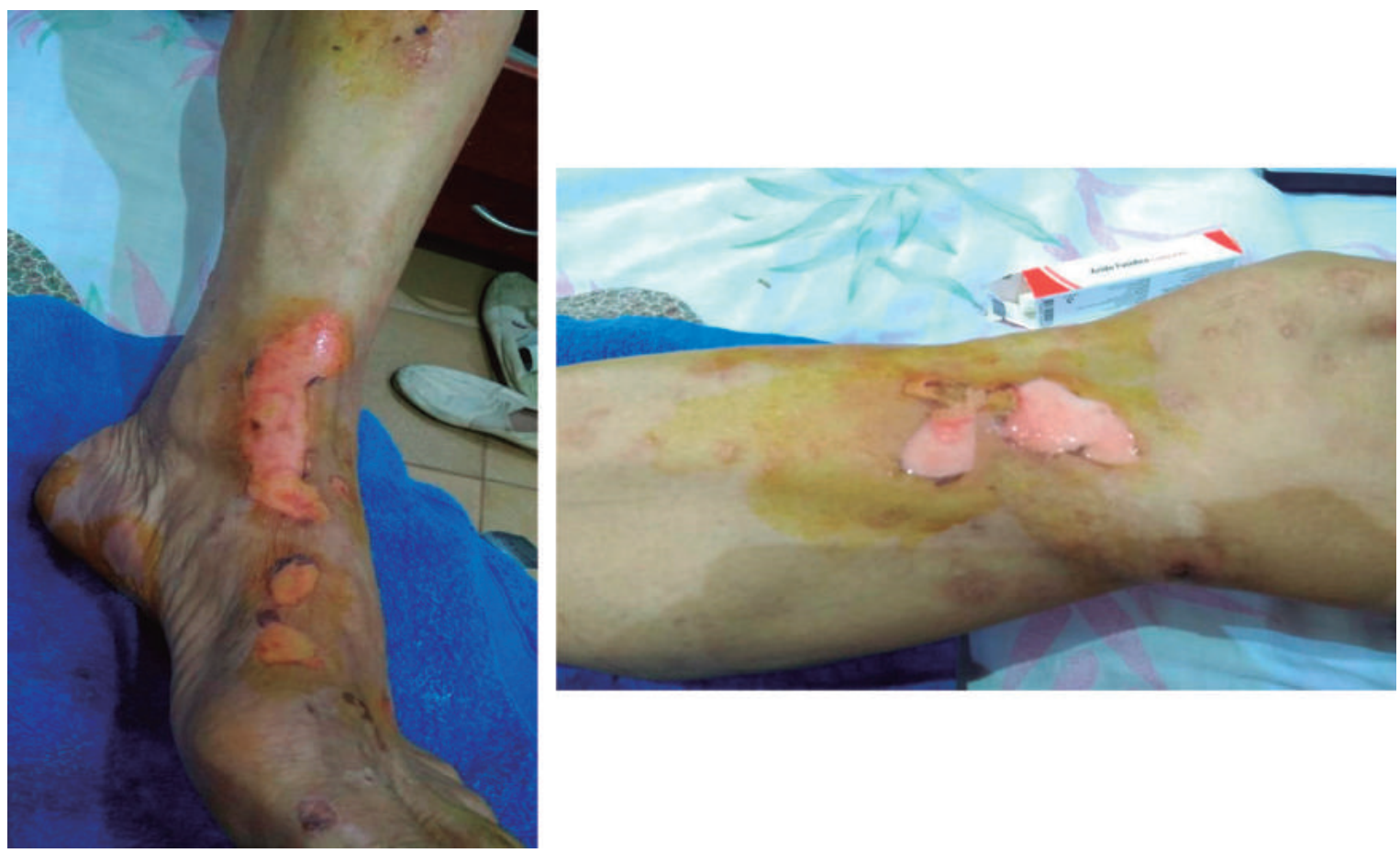
Figura 2. Caso 2. Se aprecian lesiones ampollosas en diferentes fases evolutivas hacia la mejoría.
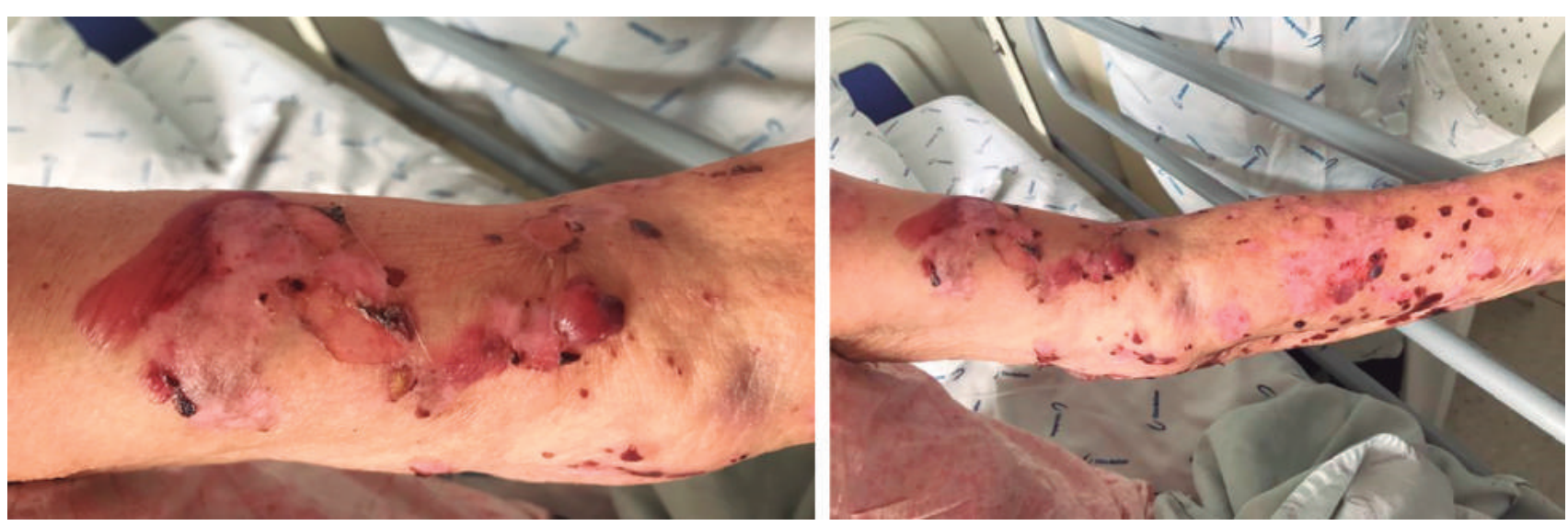

siones activas. Teniendo en cuenta lo descrito en la literatura mundial, el caso se atribuyó a la vildagliptina.

Por el compromiso extenso, el segundo caso ingresó a la unidad de cuidados intensivos (UCI) con diagnóstico de pénfigo. Recibió pulsos de metilprednisolona y posteriormente prednisona oral. Llegó a los servicios de medicina interna 12 días después, con disminución de las lesiones (Figura 2). Se planteó diagnóstico de penfigoide ampolloso asociado a vildagliptina. Dermatología por clínica planteó igual diagnóstico, que confirmó por biopsia (histopatología: ampollas subepidérmicas con numerosos infiltrados eosinófilos e inmunofluorescencia que reveló depósito lineal de inmunoglobulina $\mathrm{G}$ [IgG] en la unión dermoepidérmica). Dos meses después la paciente se encontraba asintomática.

\section{Discusión}

Las gliptinas fueron introducidas por primera vez en 2006 para tratar la DM2. Funcionan inhibiendo competitivamente la enzima DPP-IV, que normalmente descompone al péptido similar al glucagón tipo 1 (GLP-1) y al péptido insulinotrópico dependiente de la glucosa o polipéptido inhibidor gástrico (GIP). Al evitar la inactivación de GLP-1 y GIP, son capaces de aumentar la secreción de insulina y suprimir la liberación de glucagón por el páncreas (6). Comercialmente disponibles se encuentran diversos medicamentos tales como: vildagliptina, sitagliptina, saxagliptina, linagliptina, alogliptina, gemigliptina, anagliptina y teneligliptina.

Las enfermedades ampollosas autoinmunitarias se caracterizan por la presencia de ampollas y erosiones en piel, en mucosas o en ambas. Se clasifican de acuerdo con el nivel en el que ocurre la ampolla en intraepidérmicas o pénfigos y subepidérmicas o penfigoides. El diagnóstico se basa en los hallazgos clínicos, histopatológicos e inmunológicos. Estas son en- fermedades graves, dada la pérdida de la barrera cutánea, lo que conlleva riesgos de infección sobreagregada, desequilibrio hidroelectrolítico, pérdida de proteínas y muerte (7).

Desde que las gliptinas salieron al mercado se han relacionado con varias reacciones cutáneas en humanos, entre las cuales se encuentra el síndrome de Steven-Johnson (5). Se ha demostrado que la enzima DPP-IV se expresa de manera ubicua en casi todos los sistemas orgánicos, incluidos la piel y los linfocitos T (5). Aunque se ha establecido una asociación entre los iDPP-IV y el penfigoide ampolloso, la etiología exacta no ha sido claramente definida (3).

El primer informe sobre la asociación entre el uso de los inhibidores de la DPP-IV (vildagliptina) y el PA data de 2011 (4). Los casos siguientes publicados en la literatura de penfigoide ampolloso asociado a gliptinas se describen tras varios meses del inicio del tratamiento con estos medicamentos, de ahí que sea difícil en ocasiones detectar o atribuir esta reacción adversa $(3,8)$. Esta información, sumada a los resultados de los estudios de vigilancia poscomercialización de las diferentes gliptinas, ha conducido a que dicha reacción adversa sea recogida en las fichas técnicas de las presentaciones solas o en combinación y se recomiende la interrupción del tratamiento con la gliptina ante la sospecha de presencia de penfigoide ampolloso.

Hasta la fecha, se ha informado más de 60 medicamentos que inducen PA, incluidos ciertos antibióticos, diuréticos, medicamentos antihipertensivos, antifactor de necrosis tumoral alfa y vacunas (8). Sin embargo, de todas las clases de fármacos, la evidencia sólida actual sugiere que el uso previo de iDPP-IV conlleva mayor riesgo de PA, y de ahí el rápido incremento de publicaciones sobre el PA asociado al iDPP-IV (8).

Esta alerta debe tenerse en cuenta puesto que, en Colombia, según la Administradora de los Recursos del Sistema Ge- 
neral de Seguridad Social en Salud (ADRES), entre las 10 primeras prescripciones hechas a través del aplicativo MIPRES durante el año 2018 había dos iDPP-IV (Figura 3) (9).

La relevancia de estos casos no está en la presentación clínica como tal, ya que no difiere de lo publicado; sino en:

- Alertar sobre esta asociación, que no debe pasar desapercibida en la actividad médica dado el volumen de pacientes que cada vez más reciben este tipo de medicamentos.

- Los médicos no especialistas en piel siempre deben asesorarse por la especialidad respectiva ante cualquier lesión ampollosa, teniendo en cuenta que pueden equivocarse si se da pleno respaldo a la biopsia sin tener en cuenta la clínica, como sucedió con el paciente masculino. La dermatología es un campo demasiado amplio para ser abarcado por un solo experto; por eso han surgido subespecialidades como la cirugía dermatológica, la dermatología pediátrica, la oncología dermatológica y la dermatopatología.

- Hay que reconocer que la mayoría de las biopsias de piel son leídas por patólogos generales, que pueden incurrir en errores diagnósticos no intencionales y demorar así el tratamiento respectivo. Estos especialistas generalmente tienen información de las lesiones clínicas solo a través de una muestra de 3 milímetros o de una elipse de piel, hecho que ocurrió con el paciente masculino.

- Lo anterior no implica que los pacientes que usan gliptinas para la DM2 deban suspender el tratamiento o no prescribirse más. Solo hay que tener en cuenta que la asociación gliptinas y PA existe, y aunque la frecuencia va en aumento, su presentación sigue siendo poco frecuente.

Nos queda el interrogante de si la medicación con iDPP-IV por sí sola es suficiente para inducir el PA o si se requieren de otros factores no identificados todavía.

\section{Conclusión}

En conclusión, los datos epidemiológicos publicados recientemente confirman que las gliptinas son un factor importante para el desarrollo del penfigoide ampolloso (PA) en pacientes geriátricos. La larga latencia del período entre el inicio del tratamiento con una gliptina y el inicio o diagnóstico del PA debe tenerse en cuenta para que no se convierta en un factor de omisión en el diagnóstico, y así prevenir la recurrencia y los desenlaces fatales, por el bien de los pacientes presentes y futuros.

Figura 3. Top 10 de los medicamentos prescritos y suministrados en 2018 en Colombia.

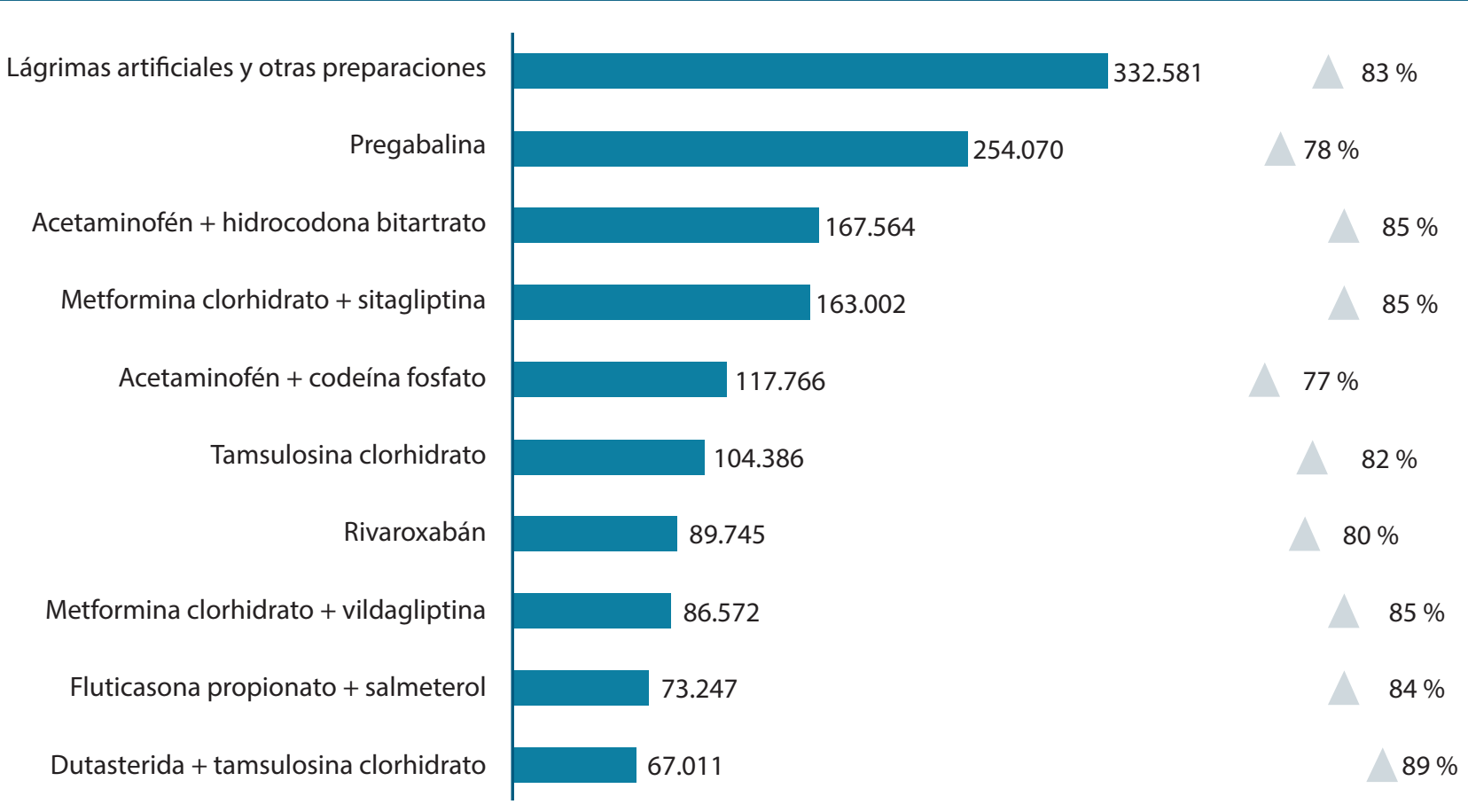




\section{Referencias}

1. Hammers CM, Stanley JR. Mechanisms of disease: pemphigus and bullous pemphigoid. Annu Rev Pathol. 2016; 11:175-97. doi: 10.1146/annurevpathol-012615-044313.

2. Kridin K. Subepidermal autoimmune bullous diseases: overview, epidemiology, and associations. Immunol Res. 2018;66(1):6-17. doi: 10.1007/ s12026-017-8975-2.

3. Kridin K, Ludwig RJ. The Growing Incidence of Bullous Pemphigoid: Overview and Potential Explanations. Front Med (Lausanne). 2018; 5:220. doi: 10.3389/fmed.2018.00220.

4. Skandalis K, Spirova M, Gaitanis G, Tsartsarakis A, Bassukas I. Drug-induced bullous pemphigoid in diabetes mellitus patients receiving dipeptidyl peptidase-IV inhibitors plus metformin. J Eur Acad Dermatol Venereol. 2012;26(2):249-53. doi: 10.1111/j.1468-3083.2011.04062.

5. Pasmatzi E, Monastirli A, Habeos J, Georgiou S, Tsambaos D. Dipeptidyl peptidase- 4 inhibitors cause bullous pemphigoid in diabetic patients: report of two cases. Diabetes Care. 2011;34(8): e133. doi:10.2337/dc110804

6. Agudelo-Zapata Y, Burgos-Cárdenas AJ, Díaz-Martínez AJ, Pinilla-Roa AE. Inhibidores de dipeptidil dipeptidasa-IV: de la teoría a la práctica. Rev Fac Med. 2015;63(2):259-70. doi: 10.15446/revfacmed. v63n2.48820.

7. Velásquez MM. Enfermedades ampollosas autoinmunitarias: pasado, presente y futuro. Rev. Asoc Colomb Dermatol. 2017;25(2):112-4.

8. Tasanen K, Varpuluoma O, Nishie W. Dipeptidyl Peptidase-4 InhibitorAssociated Bullous Pemphigoid. Front Immunol. 2019; 10:1238. doi: 10.3389/fimmu.2019.01238.

9. Recursos que se convierten en salud. Descripción de las primeras descripciones realizadas a través de MIPRES 2018 [Internet]. Administradora de los Recursos del Sistema General de Seguridad Social en Salud (ADRES). Disponible en: https://bit.ly/3qKADUw. 


\section{Que la diabetes no marque la vida de su paciente}

- La educación del paciente es parte primordial del tratamiento y es la base para que logre sus metas. ${ }^{(1)}$

- La presencia de Lipohipertrofia, reduce la absorción de insulina. La detección y manejo reducen los costos del tratamiento $^{(1)}$

- Dispositivos de calidad y un buen programa educativo son efectivos en pacientes insulinorrequirientes ${ }^{(2)}$

\section{Programa Educativo para paciente con diabetes de BD Colombia}

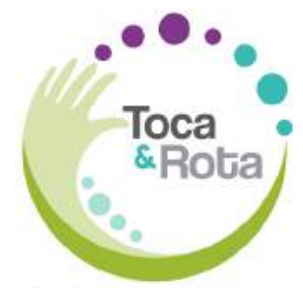

Que la diabetes no te marque, se parte y comparte.

\section{BD Ultra-Fine ${ }^{\circledR}$ Nano Easy $\underset{\text { Fechonology }}{ }$}

Aguja para Pen de insulina con pared extradelgada que ofrece mayor diámetro interno aumentando la rapidez de flujo

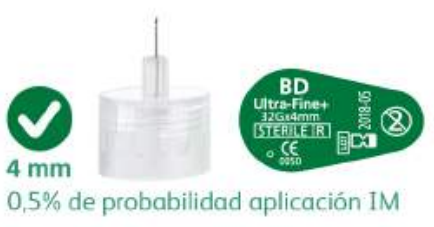

BD Ultra-fine"

Nano Penta Point ${ }^{\mathrm{N}}$ Easyflow ${ }^{\mathrm{m}}$ Aguja para PEN de insulina Caja $\times 100$ Unidades $4 m m$ - 326 Ref: 320489

INVIMA2018DM-0017818

\section{IM}
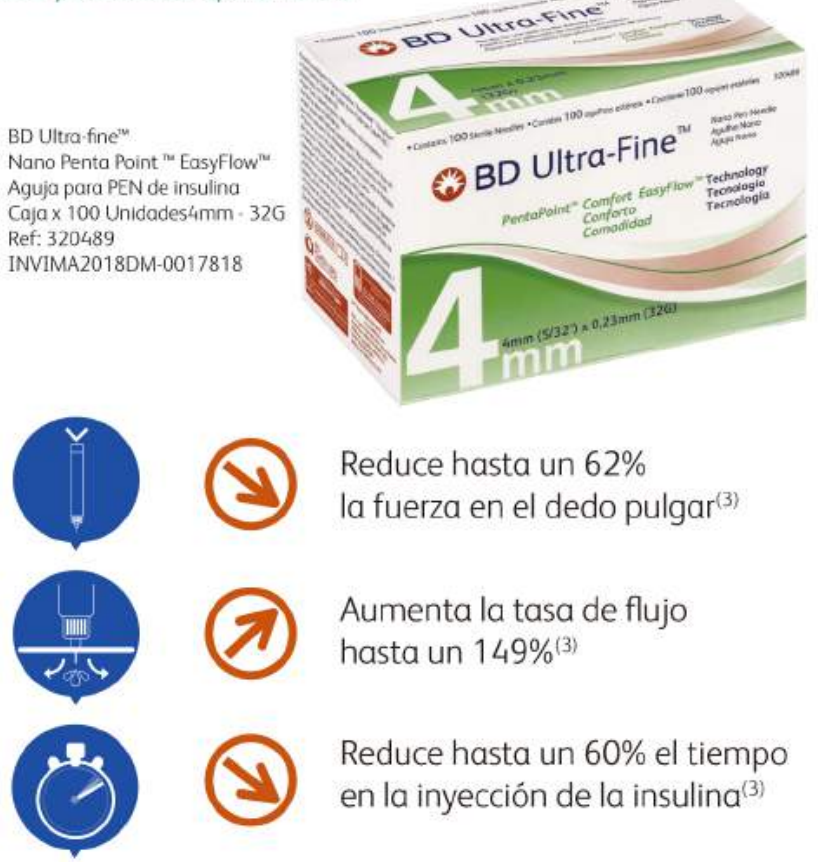

().

Reduce hasta un $62 \%$

la fuerza en el dedo pulgar ${ }^{(3)}$

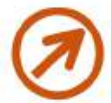

Aumenta la tasa de flujo hasta un $149 \%^{(3)}$

Reduce hasta un $60 \%$ el tiempo en la inyección de la insulina ${ }^{(3)}$

1. M. Blanco et al. / Diabetes \& Metabolism 39 (2013) 445-453

Lipohipertrofía: conocimiento y educación al tratamiento con insulina en la diabetes mellitus. Sandra Milena Cano Ibarsa, Gibria Lastre Ameli, Maria Alejandra Oróstegul Santander Revista Med 2018,26

Aronsan R, Gibney M A Oza K, Berube J, Kasler-Taub K, Hirsch L. Insulin pen needles, effects of extra-thin wall needle technoly on preference, confidence, and other patient ratings. Clincal Therapeutics 2013:35(7): 923.933 